\title{
The effect of building energy management systems on battery aging.
}

\author{
Lluc Canals Casals ${ }^{1,}{ }^{*}$, Lucía Igualada $^{1}$, and Cristina Corchero ${ }^{1}$ \\ ${ }^{1}$ Catalonia Institute for Energy Research (IREC), Energy Systems Analytics Department, 08930 Sant \\ Adrià de Besòs, Barcelona, Spain.
}

\begin{abstract}
Smart buildings are a key element to walk towards smart cities and grids. Nonetheless, there are several degrees of intelligence. A first step is to incorporate commercial self-consumption solutions in buildings so they can manage the energy from local renewable power generators. A second step is to substitute this commercial solutions with an optimized Energy Management System (EMS) to reduce the electricity bill at the end of the month. Further. This EMS may contribute to stabilize and improve the quality and emissions of the electricity grid by offering some energy flexibility to the electricity system in favour of decentralization. This study compares the battery aging between buildings that count with an EMS to optimize the electricity bill under three scenarios in contrast to those that have a simple self-consumption kit. Lithium ion battery lifespan is estimated by means of an electric equivalent battery circuit model that runs on Matlab and simulates its behaviour through time. Moreover, this study evaluates the distribution of the battery costs regarding its use, observing that batteries controlled by simple self-consumption kits have longer lifespan because they are underused, ending up in higher calendar aging costs than the ones that are controlled by EMS.
\end{abstract}

\section{Introduction}

Renewable energy sources can be integrated in any place in the electricity grid, from largescale generators to residential buildings [1]. It is widely accepted that energy storage systems such as batteries are a key element to increase the integration rate of renewable power sources into the grid [2] and both contribute on the configuration of decentralized smart grids [3]. Thus, residential buildings would be the smaller element in decentralized smart grids of this kind, being the core of this study.

To manage the energy generation and demand of buildings, several devices can be used. Generally, buildings count on self-consumption kits that use a simple energy management program, such as the one presented in Fig 1, due to the lower investment costs. These systems store the excess of energy generated by the renewable power systems of the building in the energy storage system (generally a battery). If the battery is full, this excess is delivered to the electricity grid. While the battery has enough energy and the generation

\footnotetext{
*Corresponding author: 1canals@irec.cat
} 
is lower than the building's energy demand, the battery will discharge. When the battery is completely discharged, it is the grid that should provide all the building energy demand.

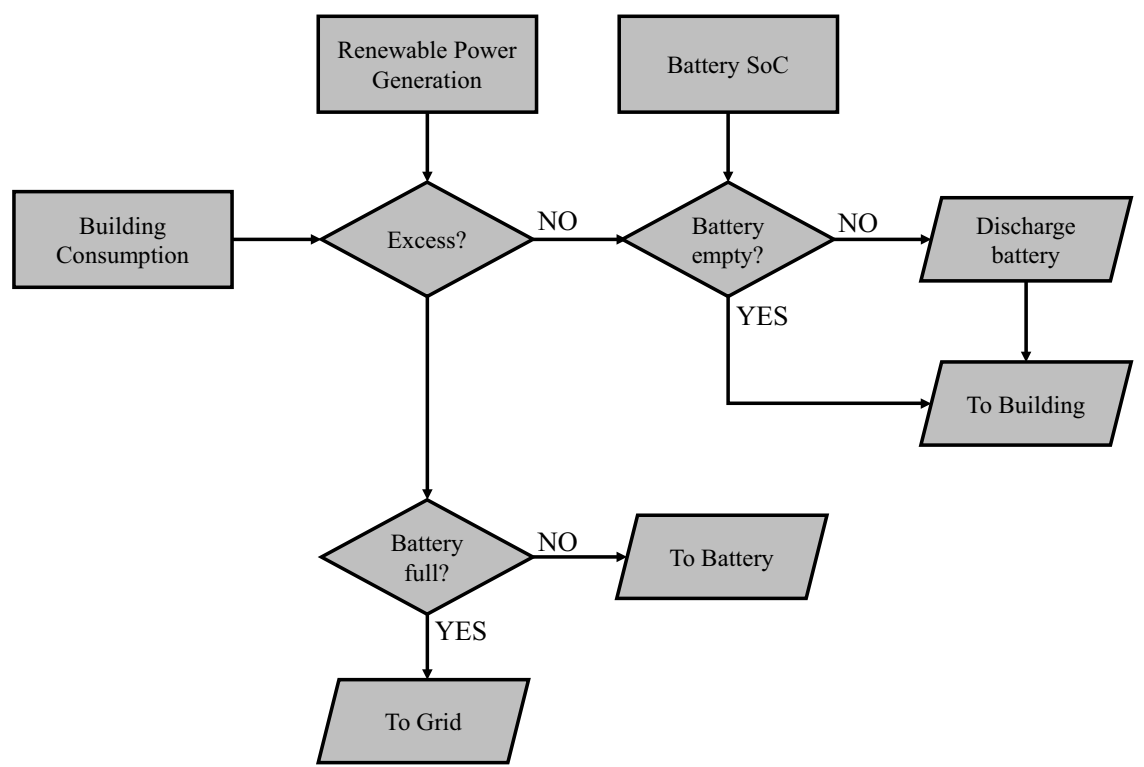

Fig 1. Example of a decision making program of a commercial self-consumption kit.

On the contrary, more complex Energy Management Systems (EMS) incorporate forecasting sources to predict weather conditions, electricity prices and building load in their decision making algorithms so they can reduce either the monthly electricity bill [4] or the greenhouse gas emissions [5].

These economically oriented EMS tend to use batteries much more often than the commercial kits do, which certainly has an impact on battery aging [6]. Although several V2G or intelligent electric vehicle charging systems do consider other aspects, such as battery lifespan enlargement, in the energy management algorithm [7], most of the algorithms implemented in households' EMS do not incorporate battery aging factors in their optimization calculations due to the complexity and particularity of ageing related to each battery chemistry.

Therefore, this study compares the effect that the different management strategies between commercial self-consumption kits and EMS under three different scenarios have on battery aging.

\section{Methodology}

This study uses monitored consumption data of the common areas (lighting, elevator, communications...) of a building during one year that is part of the GrowSmarter UE funded project. This building counts on $4 \mathrm{kWp}$ photovoltaic generators on the rooftop, a self-consumption kit and a $6,5 \mathrm{kWh}$ lithium ion battery that costs $4011 €$. The battery useful Depth of Discharge (DoD) is $90 \%$, this means that the system can use only $90 \%$ of its nominal capacity. This is the base scenario of the study in which the battery is partially used, having an average use of 0,25 cycles per day. 
A previous work [8] considered these daily generation and energy load of the building to simulate the behaviour of the EMS under three different scenarios (represented in Fig 2) and to compare the benefits it may provide against commercial systems:

- High (1): The battery is used whenever there is a positive balance in the electricity prices, doing an average of 2,4 cycles per day.

- Medium (2): The difference in price between the lower valley and the peak is higher than in the High scenario, thus, the smaller differences are not considered. In this case, the battery does over 1,5 cycles per day.

- Low (3): The battery is discharged only when the electricity peak cost is over $0,14 € / \mathrm{kWh}$ and it is charged at lower valleys. In this scenario, the battery does an average of 1 equivalent full cycle each day.

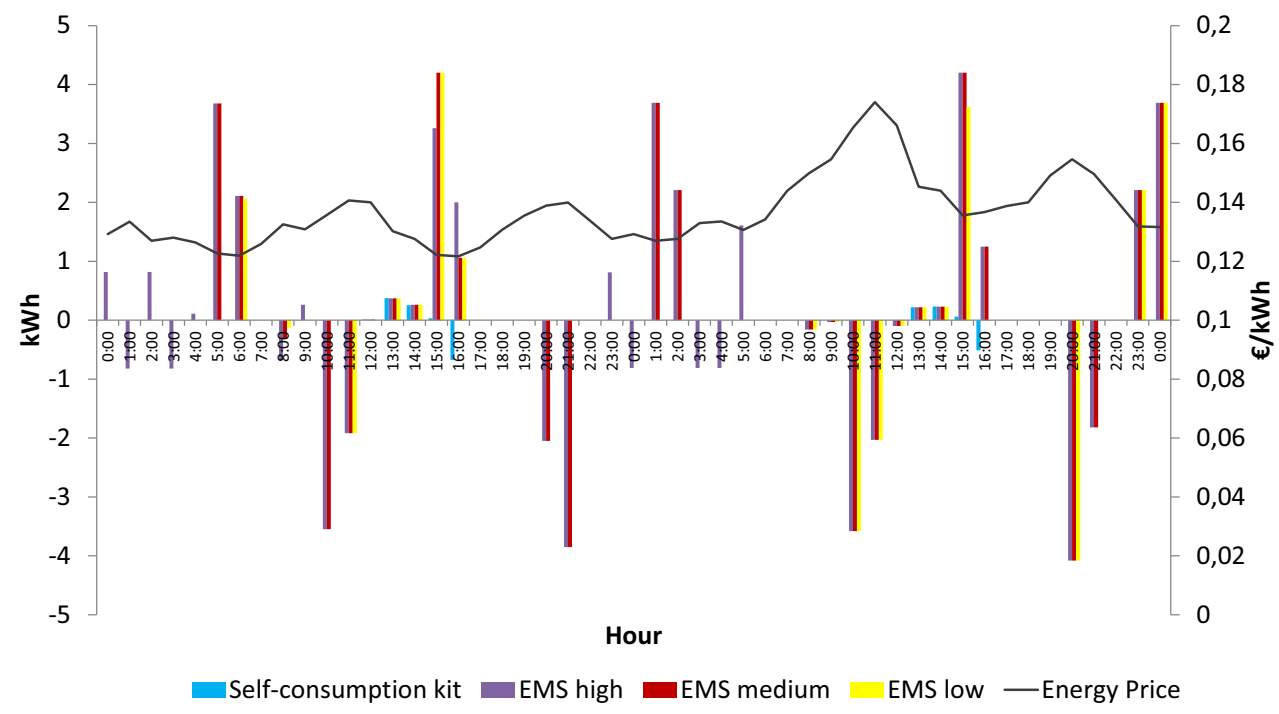

Fig 2. Representation of the battery use in all scenarios during 2 days (bars) and the electricity price (black line).

The EMS recalculates the optimal solution every 15 minutes considering inputs from the day-ahead tariff (economic), the forecasted solar energy generation, the expected battery SOC and adapting its results with the deviations occurred due to real energy consumption in the building and from what really occurs with the solar generation. Fig 2 represents the battery hourly energy exchange (bars) in all scenarios during two days extracted from the whole year monitoring and the corresponding energy price per hour (black line). Fig 2 shows how the EMS in scenario 1 (High) tries to use the battery far more often than in all the other cases. Moreover, Fig 2 shows how the commercial kit clearly underuses it and, moreover, it begins the discharge of the battery while the EMS still considers it worth charging it.

These electricity curves are then used to feed the electric equivalent battery model for each scenario. This aging model was presented and used in previous works such as[9], [10] to estimate the battery State of Health $(\mathrm{SoH})$ evolution of batteries according to their use. The End of Life (EoL) of the battery is reached when $\mathrm{SOH}$ gets to $64 \%$ of the initial battery capacity. The time the SOH needs to reach the EoL results to be the lifespan of the battery in each case. This battery aging model runs on Matlab and considers five aging factors, which are: working temperature, C-rate, DOD, State of Charge (SoC) and time. These five factors are considered to be the ones having major effect on battery aging according to 
literature [11], [12]. The $\mathrm{SoH}$ is defined as the ratio between the actual capacity of the battery against the capacity of the battery when fresh or new.

For the simulation of the battery SoH evolution, the study considered that the battery stays at a constant temperature of $25^{\circ} \mathrm{C}$.

\section{Results and discussion}

Fig 3 presents the battery aging model estimation of the $\mathrm{SoH}$ evolution along time for each scenario. These results show that, effectively, the battery lifespan using commercial kits is longer than in all the scenarios using an EMS. In fact, its lifespan is more than 3, 5 and 8 times the lifespan of a battery working with an EMS at low, medium, and high use scenarios respectively.

These results may mislead to a conclusion stating that non-intelligent energy management of commercial kits is preferable to extend the battery lifespan. Results would certainly go in the opposite direction if the EMS goal should be oriented to enlarge battery lifespan instead of economic incentives.

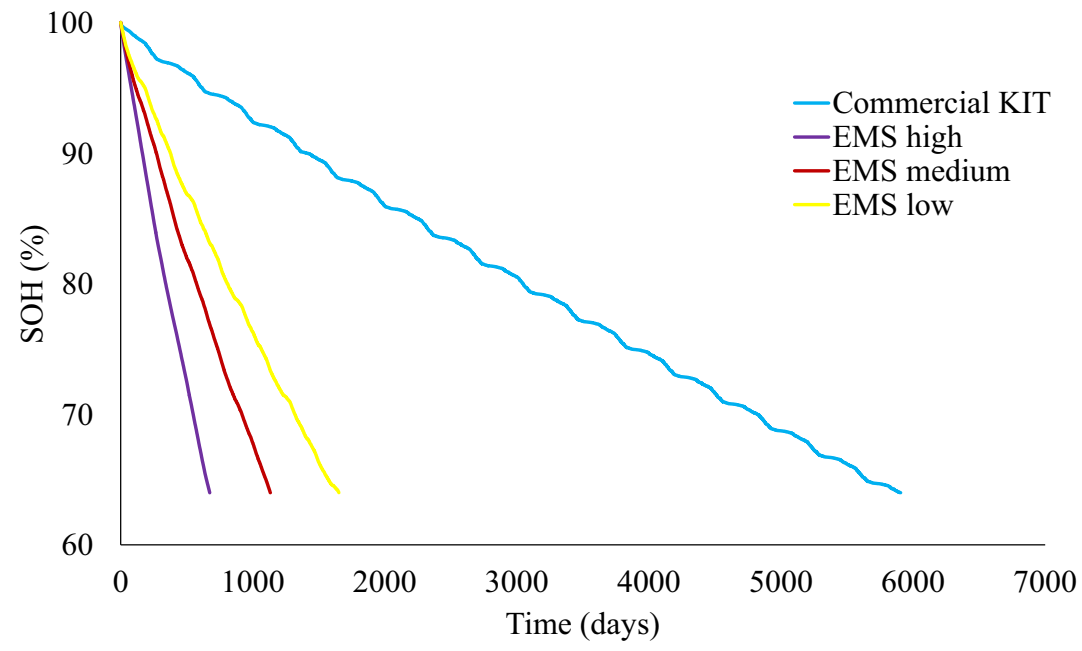

Fig 3. SoH evolution and battery lifespan for each scenario

Nonetheless, the reader would have noticed that the relation of the difference in battery lifespan is lower than the one concerning the average cycle use of the battery defined for each scenario. This difference is explained by the fact that batteries age under two circumstances, when they are under use (cycling aging) but also when they are in a standby mode (calendar aging).

The aging model used in this study for the SoH estimation considers both aging circumstances (calendar-cycling). In fact, calendar aging is affected by the battery's SoC and temperature during the stand-by lapse. Therefore, for each time-step that the battery is not used there is a loss of capacity that represents an equivalent loss of money in terms of amortization.

The proportion of battery degradation due to calendar aging is presented in Fig 4, showing that the proportion of the battery aging due to stand-by mode in the base case scenario (Kit) represents a $14 \%$ of the total aging of the battery. Translated to costs, this 
means that $521 €$ are spent just for having the battery ready without any use. In contrast, this factor is much lower for the scenarios that involve an optimized EMS.

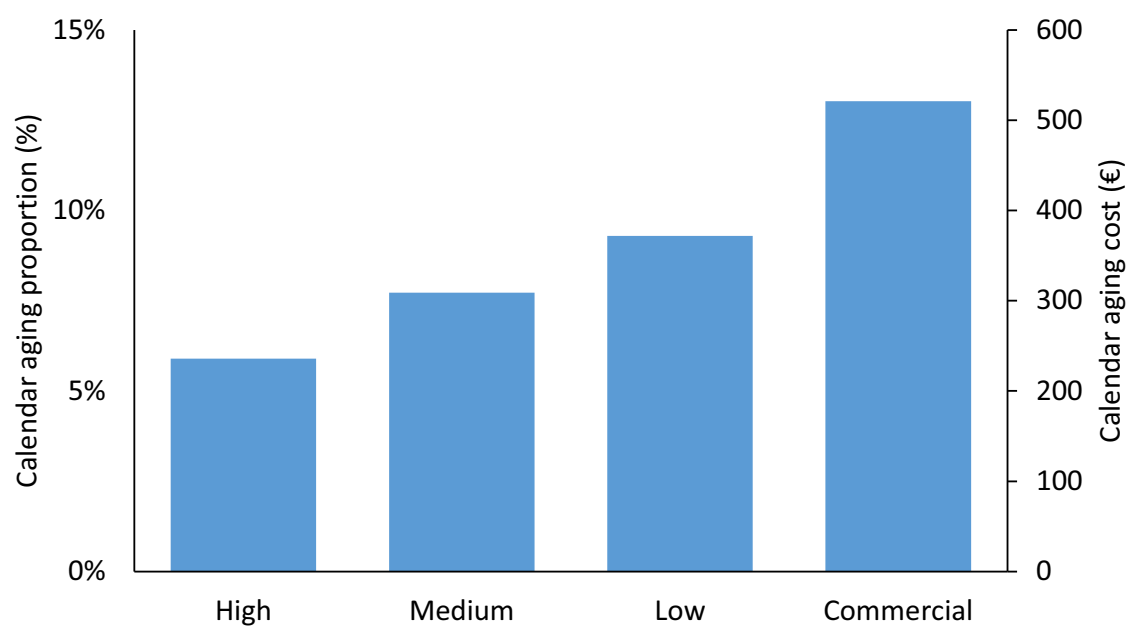

Fig 4. Battery degradation proportion due to calendar aging (left) and its attributed cost (right)

This cost analysis can also be related to the quantity of energy delivered by the battery in which case results indicate that, effectively, EMS scenarios exchange more energy than using a commercial solutions. Thus, the cost per $\mathrm{kWh}$ delivered by the battery is also lower using smart energy management systems.

Nonetheless, a thorough economic analysis should be carried out, as recent literature indicate that Li-ion batteries for stationary applications are not economically viable due to their high costs [7]. Therefore, it has sense to continue the study regarding possible incentives, energy price scenarios or the use of second life electric vehicle batteries, which have much more competitive costs [13].

\section{Conclusions}

This study analysed the behaviour of several EMS in contrast to commercial kits installed on buildings that count on power generation elements showing that buildings counting on commercial kits tend to underuse the battery capacity.

The study focus the attention on the effect of the differences in use of the battery in relation to its aging. Results show that batteries from buildings that count with an EMS oriented to optimize the electricity bill at the end of the year age faster than those related to commercial kits.

Nonetheless, the analysis indicates that the fact that commercial kits keep the battery in stand-by mode for longer periods of time reverts in a cost of opportunity that EMS manage in a better way.

However, to determine if the EMS strategy is good or not, a more thorough economic analysis should be undertaken considering several tariffs, electricity price evolutions, possible incentives and other alternatives that might decrease battery costs in the nearby future.

The research leading to these results has received funding from the European Union H2020 Programme under GrowSmarter project, Grant agreement no. 646456. 


\section{References}

[1] O. Edenhofer, R. Pichs Madruga, and Y. Sokona, Renewable Energy Sources and Climate Change Mitigation:Special Report of the Intergovernmental Panel on Climate Change (2012).

[2] B. Scrosati and J. Garche, J. Power Sources 195, 2419-2430 (2010).

[3] C. Clastres, Energy Policy 39, 5399-5408 (2011).

[4] H. Beltran, M. Swierczynski, N. Aparicio, E. Belenguer, R. Teodorescu, and P. Rodriguez, IEEE Int. Symp. Ind. Electron., 1604-1609 (2012).

[5] P. Rocha, A. Siddiqui, and M. Stadler, Energy Build. 88, 203-213 (2015).

[6] A. Barré, B. Deguilhem, S. Grolleau, M. Gérard, F. Suard, and D. Riu, J. Power Sources 241, 680-689 (2013).

[7] K. Uddin, M. Dubarry, and M. B. Glick, Energy Policy 113, 342-347 (2018).

[8] L. Igualada and C. Corchero, IV Congr. Smart Grids, (2017).

[9] L. Canals Casals, B. Amante García, and M. González Benítez, in Lect. Notes Manag. Ind. Eng. 139-151 (2017).

[10] L. Canals Casals and B. Amante García, Batteries 3(1), 10 (2017).

[11] J. Vetter, P. Nov, M. R. R. Wagner, C. Veit, P. Novák, K.-C. Möller, J. O. Besenhard, M. Winter, M. Wohlfahrt-Mehrens, et al., J. Power Sources 147, 269281 (2005).

[12] A. W. Thompson, J. Power Sources 396, 691-709 (2018).

[13] L. Canals Casals and B. Amante García, J. Green Eng. 6, 77-98 (2016). 